\title{
A SUPERCONVERGENCE RESULT FOR MIXED FINITE ELEMENT APPROXIMATIONS OF THE EIGENVALUE PROBLEM *
}

\author{
QUN $\operatorname{Lin}^{1}$ AND HeHU XIE ${ }^{1}$
}

\begin{abstract}
In this paper, we present a superconvergence result for the mixed finite element approximations of general second order elliptic eigenvalue problems. It is known that a superconvergence result has been given by Durán et al. [Math. Models Methods Appl. Sci. 9 (1999) 1165-1178] and Gardini [ESAIM: M2AN 43 (2009) 853-865] for the lowest order Raviart-Thomas approximation of Laplace eigenvalue problems. In this work, we introduce a new way to derive the superconvergence of general second order elliptic eigenvalue problems by general mixed finite element methods which have the commuting diagram property. Some numerical experiments are given to confirm the theoretical analysis.
\end{abstract}

Mathematics Subject Classification. 65N30, 65N25, 65L15, 65B99.

Received September 26, 2010. Revised July 22, 2011.

Published online February 3, 2012.

\section{INTRODUCTION}

In this paper, we are concerned with the following second order elliptic eigenvalue problem: find $(p, \lambda)$ such that

$$
\left\{\begin{aligned}
-\nabla \cdot(\mathcal{A} \nabla p)+\varphi p & =\lambda \rho p, & & \text { in } \Omega, \\
\mathcal{B}(p) & =0, & & \text { on } \partial \Omega, \\
\int_{\Omega} \rho p^{2} \mathrm{~d} \Omega & =1, & &
\end{aligned}\right.
$$

where $\mathcal{A}=\left(a_{i j}\right)_{2 \times 2}$ is a symmetric positive definite matrix with $a_{i j} \in W^{1, \infty}(\Omega)$ for $1 \leq i, j \leq 2,0 \leq \varphi \in$ $W^{0, \infty}(\Omega)$ on $\bar{\Omega}, \rho$ is a bounded positive function on $\bar{\Omega}$ and $0<c_{0} \leq \rho \in W^{0, \infty}(\Omega), \Omega \subset \mathcal{R}^{2}$ is a bounded domain with Lipschitz boundary $\partial \Omega, \nabla$ and $\nabla$. denote the gradient and divergence operators and $\mathcal{B}(p)$ denotes the boundary condition which can be Dirichlet or Neumann type, i.e.,

$$
\mathcal{B}_{D}(p)=p=0, \quad \text { on } \partial \Omega,
$$

or

$$
\mathcal{B}_{N}(p)=\mathbf{n} \cdot \mathcal{A} \nabla p=0, \quad \text { on } \partial \Omega .
$$

Keywords and phrases. Second order elliptic eigenvalue problem, mixed finite element method, superconvergence.

* This project was supported by the National Natural Science Foundation of China (11001259, 11031006, 2011CB309703).

1 LSEC, Institute of Computational Mathematics, Academy of Mathematics and Systems Science, Chinese Academy of Sciences, Beijing 100190, P.R. China. linq@lsec.cc.ac.cn; hhxie@lsec.cc.ac.cn 
The mixed formulation for the eigenvalue problem (1.1) comes from computing the vibration modes of a fluid in a displacement formulations since using the displacement formulation for fluid is more convenient than using the pressure or potential as variable (see, e.g., [11]).

There are several works for the second order elliptic eigenvalue problems in the mixed formulation and their numerical methods such as Babuška and Osborn [1,2,21], Mercier et al. [20], Boffi et al. [5,6], Boffi [4], etc. Osborn [21], Mercier et al. [20], Boffi et al. [5], Boffi [4] give the analysis for the eigenpair approximations by mixed/hybrid finite element methods based on the theory of compact operator (see, e.g., [9]).

In [11-13], a superconvergence result between the lowest order Raviart-Thomas mixed finite element approximation and their corresponding mixed finite element projection for the Laplace eigenvalue problem with $\mathcal{A}=I$, $\varphi=0$ and $\rho=1$ in (1.1) has been proven. Their analysis is based on the equivalence between the lowest order Raviart-Thomas approximation and the non-conforming Crouzeix-Raviart approximation for Laplace eigenvalue problem with Neumann boundary condition. In this paper, we extend the superconvergence result to general second order elliptic eigenvalue problems with general mixed finite element methods. This is also an extension of the superconvergence for the second order elliptic problem by mixed finite element methods (see, e.g., [8]).

The outline of the paper goes as follows. In Section 2, we introduce some preliminaries and notations, and state the weak form of the eigenvalue problem and its corresponding discrete form. A superconvergence result between the eigenfunction approximations and its corresponding interpolant is obtained in Section 3. Some numerical results are given in Section 4 to confirm the theoretical analysis in Section 3. Some concluding remarks are given in the last section.

Throughout this paper $C$ or $c$, denotes a generic positive constant which is independent of the mesh size but sometimes depends on the eigenvalues of the problem (1.1).

\section{Mixed Finite ELEMENT METHOD}

We define a new vector-valued function $\mathbf{u}$ as follows

$$
\mathbf{u}=\mathcal{A} \nabla p
$$

Then (1.1) can be transformed into the following equivalent mixed formulation

$$
\left\{\begin{aligned}
-\nabla \cdot \mathbf{u}+\varphi p & =\lambda \rho p, & & \text { in } \Omega, \\
\mathcal{A}^{-1} \mathbf{u}-\nabla p & =0, & & \text { in } \Omega, \\
\int_{\Omega} \rho p^{2} \mathrm{~d} \Omega & =1, & &
\end{aligned}\right.
$$

with the boundary condition

$$
p=0, \quad \text { on } \partial \Omega,
$$

for the Dirichlet boundary case (1.2) or

$$
\mathbf{n} \cdot \mathbf{u}=0, \quad \text { on } \partial \Omega,
$$

for the Neumann boundary case (1.3).

Let $W:=L^{2}(\Omega)$ be the standard $L^{2}$ space on $\Omega$ with norm $\|\cdot\|_{0}$ and let $\mathbf{V}$ be the Hilbert space

$$
\mathbf{V}:=H(\operatorname{div}, \Omega)=\left\{\mathbf{v} \in\left(L^{2}(\Omega)\right)^{2}: \nabla \cdot \mathbf{v} \in L^{2}(\Omega)\right\}
$$

for Dirichlet boundary condition (1.2) or

$$
\mathbf{V}:=H_{0}(\operatorname{div}, \Omega)=\left\{\mathbf{v} \in\left(L^{2}(\Omega)\right)^{2}: \nabla \cdot \mathbf{v} \in L^{2}(\Omega), \mathbf{n} \cdot \mathbf{v}=0\right\}
$$


for Neumann boundary condition (1.3) equipped with the norm

$$
\|\mathbf{v}\|_{\mathbf{v}}:=\left(\|\mathbf{v}\|_{0}^{2}+\|\nabla \cdot \mathbf{v}\|_{0}^{2}\right)^{\frac{1}{2}}
$$

respectively.

The corresponding weak formulation for the problem (2.1) seeks $(\mathbf{u}, p, \lambda) \in \mathbf{V} \times W \times \mathcal{R}$ such that $p \neq 0$ and

$$
\left\{\begin{array}{rlrl}
a(\mathbf{u}, \mathbf{v})-b(\mathbf{v}, p) & =0, & & \forall \mathbf{v} \in \mathbf{V}, \\
b(\mathbf{u}, q)+d(p, q) & =\lambda r(p, q), & \forall q \in W, \\
r(p, p) & =1, & &
\end{array}\right.
$$

where $a(\cdot, \cdot), b(\cdot, \cdot), d(\cdot, \cdot)$ and $r(\cdot, \cdot)$ are bilinear forms defined by

$$
\begin{gathered}
a(\mathbf{u}, \mathbf{v})=\int_{\Omega} \mathbf{u} \cdot \mathcal{A}^{-1} \mathbf{v} \mathrm{d} \Omega, \quad b(\mathbf{v}, q)=-\int_{\Omega} \nabla \cdot \mathbf{v} q \mathrm{~d} \Omega, \\
d(p, q)=\int_{\Omega} \varphi p q \mathrm{~d} \Omega, \quad r(p, q)=\int_{\Omega} \rho p q \mathrm{~d} \Omega .
\end{gathered}
$$

For the aim of the analysis, we also need to define the weighted $L^{2}(\Omega)$ norm based on the inner product $r(\cdot, \cdot)$ as

$$
\|q\|_{r}:=r(q, q)^{\frac{1}{2}} .
$$

Based on the property of the function $\rho$, it is obvious that the norm $\|\cdot\|_{r}$ is equivalent to $\|\cdot\|_{0}$.

From [2], we know that the eigenvalue problem (2.4) owns an eigenvalue sequence $\left\{\lambda_{j}\right\}$ :

$$
0 \leq \lambda_{1} \leq \lambda_{2} \leq \ldots \leq \lambda_{k} \leq \ldots, \quad \lim _{k \rightarrow \infty} \lambda_{k}=\infty
$$

and the associated eigenfunctions

$$
\left(\mathbf{u}_{1}, p_{1}\right),\left(\mathbf{u}_{2}, p_{2}\right), \ldots,\left(\mathbf{u}_{k}, p_{k}\right), \ldots
$$

where $r\left(p_{i}, p_{j}\right)=\delta_{i j}$.

Now, let us define the mixed finite element discretization method for the eigenvalue problem (2.4). The wellposedness of the discrete weak form of (2.4) can be guaranteed by the fact that the corresponding approximation spaces satisfy the Babuška-Brezzi condition (see, e.g., $[7,8,14]$ ). Let $\mathcal{T}_{h}$ be a partition of $\Omega$ into finite elements (triangles or rectangles), which is regular and has a mesh size $h$. Associated with the partition $\mathcal{T}_{h}$, we define the finite dimensional spaces $\mathbf{V}_{h} \subset \mathbf{V}$ and $W_{h} \subset W$ as finite element spaces [8,14].

In this paper, we assume the mixed finite element space $\mathbf{V}_{h} \times W_{h}$ has an interpolation operator $\left(\mathbf{I}_{h}, J_{h}\right)$

$$
\mathbf{I}_{h} \times J_{h}: \mathbf{V} \times W \longmapsto \mathbf{V}_{h} \times W_{h}
$$

satisfying the following commuting diagram property (see, e.g., [8])

$$
\nabla \cdot \mathbf{I}_{h} \mathbf{v}=J_{h} \nabla \cdot \mathbf{v}, \quad \forall \mathbf{v} \in \mathbf{V} \cap H^{\epsilon}(\Omega),
$$

where $J_{h}$ denotes the $L^{2}$-projection on $W_{h}$.

The mixed finite element space $\mathbf{V}_{h} \times W_{h}$ is assumed to have the following approximation properties:

$$
\begin{aligned}
\left\|\mathbf{I}_{h} \mathbf{v}\right\|_{0} & \leq C\|\mathbf{v}\|_{1}, \\
\left\|\mathbf{u}-\mathbf{I}_{h} \mathbf{u}\right\|_{\mathbf{v}}+\left\|p-J_{h} p\right\|_{0} & \leq C h^{m}\left(\|\mathbf{u}\|_{m}+\|\nabla \cdot \mathbf{u}\|_{m}+\|p\|_{m}\right), \quad 0<m \leq k,
\end{aligned}
$$

for any $\mathbf{u} \in\left(H^{m}(\Omega)\right)^{2}, \nabla \cdot \mathbf{u} \in H^{m}(\Omega)$, and $p \in H^{m}(\Omega)$, where $k$ is a positive integer according to the degree of the mixed finite element space $\mathbf{V}_{h} \times W_{h}$. 
Now, let us define the approximation of eigenpair $(\mathbf{u}, p, \lambda)$ of $(2.4)$ by the mixed finite element method as finding a pair of $\left(\mathbf{u}_{h}, p_{h}, \lambda_{h}\right) \in \mathbf{V}_{h} \times W_{h} \times \mathcal{R}$ such that

$$
\left\{\begin{aligned}
a\left(\mathbf{u}_{h}, \mathbf{v}_{h}\right)-b\left(\mathbf{v}_{h}, p_{h}\right) & =0, & & \forall \mathbf{v}_{h} \in \mathbf{V}_{h} \\
b\left(\mathbf{u}_{h}, q_{h}\right)+d\left(p_{h}, q_{h}\right) & =\lambda_{h} r\left(p_{h}, q_{h}\right), & & \forall q_{h} \in W_{h}, \\
r\left(p_{h}, p_{h}\right) & =1 . & &
\end{aligned}\right.
$$

In this paper, we assume the eigenfunctions of (1.1) has the regularity $p \in H^{1+t}(\Omega)$, i.e.,

$$
\|p\|_{1+t} \leq C,
$$

where $t$ is a positive real number and the constant $C$ depends on the eigenfunction $p$. From [2] the discrete eigenvalue problem (2.8) has eigenvalues

$$
0 \leq \lambda_{1, h} \leq \lambda_{2, h} \leq \ldots \leq \lambda_{k, h} \leq \ldots \leq \lambda_{N, h},
$$

and the corresponding eigenfunctions

$$
\left(\mathbf{u}_{1, h}, p_{1, h}\right),\left(\mathbf{u}_{2, h}, p_{2, h}\right), \ldots,\left(\mathbf{u}_{k, h}, p_{k, h}\right), \ldots,\left(\mathbf{u}_{N, h}, p_{N, h}\right),
$$

where $r\left(p_{i, h}, p_{j, h}\right)=\delta_{i j}, 1 \leq i, j \leq N\left(N\right.$ is the dimension of the mixed finite element space $\left.\mathbf{V}_{h} \times W_{h}\right)$. For simplicity, we only consider numerical approximations of simple eigenvalues in this paper.

\section{A superCONVERGENCE RESUlT}

In [11-13], a type of superconvergence between the eigenfunction approximation and its corresponding mixed finite element projection has been given for Laplace eigenvalue problems $(\mathcal{A}=I, \varphi=0$ and $\rho=1)$ by the lowest order Raviart-Thomas mixed finite element. For the general second order elliptic eigenvalue problem (1.1), there is no corresponding superconvergence result. In this section, we show the same superconvergence result for the general eigenvalue problem (1.1) with general mixed finite elements which satisfy the commuting diagram property $(2.5)$.

In order to deduce the superconvergence result, we need to define the solution operators: $T$ and $S$, and their discrete version $T_{h}$ and $S_{h}$.

First, let us define the pair of solution operators $T$ and $S$ as

$$
T: W \longmapsto W, \quad S: W \longmapsto \mathbf{V}
$$

such that for any $g \in W$

$$
\left\{\begin{aligned}
a(S g, \mathbf{v})-b(\mathbf{v}, T g) & =0, & & \forall \mathbf{v} \in \mathbf{V} \\
b(S g, q)+d(T g, q) & =r(g, q), & & \forall q \in W
\end{aligned}\right.
$$

For this elliptic problem, the following regularity estimate holds (see, e.g., [3, 15])

$$
\|S g\|_{\gamma}+\|T g\|_{1+\gamma} \leq C\|g\|_{0},
$$

where $\gamma \in(0,1]$ depends on the maximum interior angle $\omega<2 \pi$ of $\Omega$ (for example, $\gamma=\frac{\pi}{\omega}-\epsilon$ and $\gamma=1$ if $\Omega$ is convex for the Dirichlet boundary condition (1.2) case).

Then we define the corresponding discrete pair of operators $T_{h}$ and $S_{h}$

$$
T_{h}: W \longmapsto W_{h}, \quad S_{h}: W \longmapsto \mathbf{V}_{h}
$$

such that for any $g \in W$

$$
\left\{\begin{aligned}
a\left(S_{h} g, \mathbf{v}_{h}\right)-b\left(\mathbf{v}_{h}, T_{h} g\right) & =0, & & \forall \mathbf{v}_{h} \in \mathbf{V}_{h} \\
b\left(S_{h} g, q_{h}\right)+d\left(T_{h} g, q_{h}\right) & =r\left(g, q_{h}\right), & & \forall q_{h} \in W_{h}
\end{aligned}\right.
$$


Based on the Babuška-Brezzi condition of the mixed finite element space $\mathbf{V}_{h} \times W_{h}$, we have

$$
\left\|S_{h} g\right\|_{\mathbf{V}}+\left\|T_{h} g\right\|_{0} \leq C\|g\|_{0} .
$$

We also need to define the mixed finite element projection operator $\left(R_{h}, G_{h}\right)$ by

$$
R_{h} \times G_{h}: W \times \mathbf{V} \longmapsto W_{h} \times \mathbf{V}_{h}
$$

such that for $(p, \mathbf{u}) \in W \times \mathbf{V}$

$$
\left\{\begin{aligned}
a\left(G_{h}(\mathbf{u}, p), \mathbf{v}_{h}\right)-b\left(\mathbf{v}_{h}, R_{h}(\mathbf{u}, p)\right) & =a\left(\mathbf{u}, \mathbf{v}_{h}\right)-b\left(\mathbf{v}_{h}, p\right), & & \forall \mathbf{v}_{h} \in \mathbf{V}_{h} \\
b\left(G_{h}(\mathbf{u}, p), q_{h}\right)+d\left(R_{h}(\mathbf{u}, p), q_{h}\right) & =b\left(\mathbf{u}, q_{h}\right)+d\left(p, q_{h}\right), & & \forall q_{h} \in W_{h} .
\end{aligned}\right.
$$

Notice that operator $G_{h}$ and $R_{h}$ are mutually coupled through system (3.5). For this type of projection, we have the following error estimate (see, e.g., [8])

$$
\left\|G_{h}(\mathbf{u}, p)-\mathbf{u}\right\|_{\mathbf{v}}+\left\|R_{h}(\mathbf{u}, p)-p\right\|_{0} \leq C h^{s},
$$

where $(\mathbf{u}, p)$ satisfies problem (2.1) and the constant $C$ depends on the eigenfunction $(\mathbf{u}, p)$ but independent of the mesh size $h$.

So the eigenvalue problem (2.1) can be written as

$$
\lambda T p=p,
$$

and the discrete eigenvalue problem (2.8) can also be written as

$$
\lambda_{h} T_{h} p_{h}=p_{h} .
$$

Based on the operator definitions (3.1), (3.3), and (3.5), the following relation holds

$$
R_{h}(S, T)=T_{h},
$$

where the composite operator $R_{h}(S, T)$ is defined by

$$
R_{h}(S, T) g=R_{h}(S g, T g), \quad \forall g \in W,
$$

and this type of definition is also valid for $G_{h}(S, T)$. From the projection definition (3.5) and equations (3.7) and (3.8), we also have the following relations

$$
\lambda_{h} R_{h}(S, T) p_{h}=p_{h},
$$

and

$$
\lambda R_{h}(S, T) p=R_{h}(\mathbf{u}, p) .
$$

Throughout this paper, we assume the following hypothesis holds

$$
\left\|S g-\mathbf{I}_{h} S g\right\|_{0} \rightarrow 0, \quad \text { as } h \rightarrow 0 \text { for any } g \in L^{2}(\Omega) .
$$

Based on the abstract theory of $[2,20]$, the recent important results $[4,5]$, and [6], Lemma 6.1, and (2.6), (2.7) and (3.12), we know a priori error estimates for the eigenpair approximation $\left(\mathbf{u}_{h}, p_{h}, \lambda_{h}\right)$ of $(2.8)$ when $h$ is small enough,

$$
\left\{\begin{array}{l}
\left|\lambda-\lambda_{h}\right| \leq C h^{2 s} \\
\left\|\mathbf{u}-\mathbf{u}_{h}\right\|_{\mathbf{v}}+\left\|p-p_{h}\right\|_{0} \leq C h^{s}
\end{array}\right.
$$

where $s=\min \{k, t\}$.

For the aim of the superconvergence analysis for the eigenfunction projection $R_{h}(\mathbf{u}, p)$ and the eigenfunction approximation $p_{h}$, we need the following superconvergence between $J_{h} p$ and $R_{h}(\mathbf{u}, p)$ which has been analyzed by Douglas and Roberts in 1985 (see [10]) and Brezzi and Fortin in 1991 (see [8]). 
Lemma 3.1 (cf. [8]). Assume the mixed finite element space $\mathbf{V}_{h} \times W_{h}$ satisfy the commuting diagram (2.5). We have the following superconvergence result for the mixed finite element projection $R_{h}(\mathbf{u}, p)$ and the corresponding interpolant $J_{h} p$

$$
\left\|R_{h}(\mathbf{u}, p)-J_{h} p\right\|_{0} \leq C h^{s+\gamma} .
$$

Proof. First we choose a function $\phi$ such that $\|\phi\|_{r}=1$ and $\left\|R_{h}(\mathbf{u}, p)-J_{h} p\right\|_{r}=r\left(R_{h}(\mathbf{u}, p)-J_{h} p, \phi\right)$. Then we define the auxiliary equation:

Find $(\mathbf{z}, \psi) \in \mathbf{V} \times W$ such that

$$
\left\{\begin{aligned}
a(\mathbf{v}, \mathbf{z})-b(\mathbf{v}, \psi) & =0, & & \forall \mathbf{v} \in \mathbf{V}, \\
b(\mathbf{z}, q)+d(q, \psi) & =r(q, \phi), & & \forall q \in W .
\end{aligned}\right.
$$

This auxiliary equation has the following regularity $([3,15])$

$$
\|\mathbf{z}\|_{\gamma}+\|\psi\|_{1+\gamma} \leq C\|\phi\|_{r} .
$$

The corresponding mixed finite element approximation $\left(\mathbf{z}_{h}, \psi_{h}\right) \in \mathbf{V}_{h} \times W_{h}$ is defined as:

$$
\left\{\begin{aligned}
a\left(\mathbf{v}_{h}, \mathbf{z}_{h}\right)-b\left(\mathbf{v}_{h}, \psi_{h}\right) & =0, & & \forall \mathbf{v}_{h} \in \mathbf{V}_{h}, \\
b\left(\mathbf{z}_{h}, q_{h}\right)+d\left(q_{h}, \psi_{h}\right) & =r\left(q_{h}, \phi\right), & & \forall q_{h} \in W_{h} .
\end{aligned}\right.
$$

The approximation $\left(\mathbf{z}_{h}, \psi_{h}\right)$ has the following error estimate

$$
\left\|\mathbf{z}-\mathbf{z}_{h}\right\|_{0}+\left\|\psi-\psi_{h}\right\|_{0} \leq C h^{\gamma}\|\phi\|_{r} .
$$

Then with commuting diagram property (2.5) and equations (3.5), (3.15), (3.17), we have

$$
\begin{aligned}
\left\|R_{h}(\mathbf{u}, p)-J_{h} p\right\|_{r}= & r\left(R_{h}(\mathbf{u}, p)-J_{h} p, \phi\right) \\
= & b\left(\mathbf{z}_{h}, R_{h}(\mathbf{u}, p)-J_{h} p\right)+d\left(R_{h}(\mathbf{u}, p)-J_{h} p, \psi_{h}\right) \\
= & b\left(\mathbf{z}_{h}, R_{h}(\mathbf{u}, p)-p\right)+d\left(R_{h}(\mathbf{u}, p)-J_{h} p, \psi_{h}\right) \\
= & a\left(G_{h}(\mathbf{u}, p)-\mathbf{u}, \mathbf{z}_{h}\right)+d\left(R_{h}(\mathbf{u}, p)-J_{h} p, \psi_{h}\right) \\
= & a\left(G_{h}(\mathbf{u}, p)-\mathbf{u}, \mathbf{z}_{h}-\mathbf{z}\right)+a\left(G_{h}(\mathbf{u}, p)-\mathbf{u}, \mathbf{z}\right)+d\left(R_{h}(\mathbf{u}, p)-J_{h} p, \psi_{h}\right) \\
= & a\left(G_{h}(\mathbf{u}, p)-\mathbf{u}, \mathbf{z}_{h}-\mathbf{z}\right)+b\left(G_{h}(\mathbf{u}, p)-\mathbf{u}, \psi\right)+d\left(R_{h}(\mathbf{u}, p)-J_{h} p, \psi_{h}\right) \\
= & a\left(G_{h}(\mathbf{u}, p)-\mathbf{u}, \mathbf{z}_{h}-\mathbf{z}\right)+b\left(G_{h}(\mathbf{u}, p)-\mathbf{u}, \psi-\psi_{h}\right)+b\left(G_{h}(\mathbf{u}, p)-\mathbf{u}, \psi_{h}\right) \\
& +d\left(R_{h}(\mathbf{u}, p)-p, \psi_{h}\right)+d\left(p-J_{h} p, \psi_{h}\right) \\
= & a\left(G_{h}(\mathbf{u}, p)-\mathbf{u}, \mathbf{z}_{h}-\mathbf{z}\right)+b\left(G_{h}(\mathbf{u}, p)-\mathbf{u}, \psi-\psi_{h}\right)+d\left(p-J_{h} p, \psi_{h}\right) .
\end{aligned}
$$

From (3.6) and (3.18), it is easy to know that

$$
\begin{aligned}
\left|a\left(G_{h}(\mathbf{u}, p)-\mathbf{u}, \mathbf{z}_{h}-\mathbf{z}\right)\right| & \leq C h^{s+\gamma}, \\
\left|b\left(G_{h}(\mathbf{u}, p)-\mathbf{u}, \psi-\psi_{h}\right)\right| & \leq C h^{s+\gamma} .
\end{aligned}
$$

We can also estimate the term $d\left(p-J_{h} p, \psi_{h}\right)$ as

$$
\begin{aligned}
\left|d\left(p-J_{h} p, \psi_{h}\right)\right| & \leq\left|\int_{\Omega} \bar{\varphi}\left(p-J_{h} p\right) \psi_{h} \mathrm{~d} \Omega\right|+\left|\int_{\Omega}(\varphi-\bar{\varphi})\left(p-J_{h} p\right) \psi_{h} \mathrm{~d} \Omega\right| \\
& \leq C h^{s+1}\|\varphi\|_{1},
\end{aligned}
$$

where $\bar{\varphi}$ is the interplant of $\varphi$ onto the piecewise constant function. Combining (3.19), (3.20), (3.21), and (3.22), we can obtain

$$
\left\|R_{h}(\mathbf{u}, p)-J_{h} p\right\|_{r} \leq C h^{s+\gamma} .
$$

Combining the above inequality with the equivalence of norms $\|\cdot\|_{0}$ and $\|\cdot\|_{r}$, the desired result (3.14) can be derived. 
Now, let us state the superconvergence result which is the main content in this section. The idea we use here comes from [18] which is based on the operator analysis.

Theorem 3.2. Let $\left(\mathbf{u}_{h}, p_{h}, \lambda_{h}\right)$ be the corresponding discrete eigenpair approximation for the eigenvalue problem (2.8) and $\left(G_{h}(\mathbf{u}, p), R_{h}(\mathbf{u}, p)\right)$ be the mixed finite element projection of $(\mathbf{u}, p)$ defined by (3.5) where $(\mathbf{u}, p)$ is the solution of (2.1). When the mesh size $h$ is small enough, we have the following superconvergence result

$$
\left\|R_{h}(\mathbf{u}, p)-p_{h}\right\|_{0} \leq C h^{s+\gamma},
$$

where the constant $C$ depends on the eigenvalue $\lambda$ but independent of the mesh size $h$.

Proof. First from (3.7), (3.10), and (3.11), we have

$$
\begin{aligned}
(I-\lambda T)\left(R_{h}(\mathbf{u}, p)-p_{h}\right)= & \left(\lambda_{h} R_{h}(S, T)-\lambda T\right)\left(R_{h}(\mathbf{u}, p)-p_{h}\right)+R_{h}(\mathbf{u}, p)-p_{h} \\
& -\lambda_{h} R_{h}(S, T)\left(R_{h}(\mathbf{u}, p)-p\right)-\lambda_{h} R_{h}(S, T)\left(p-p_{h}\right) \\
= & \left(\lambda_{h} R_{h}(S, T)-\lambda T\right)\left(R_{h}(\mathbf{u}, p)-p_{h}\right)+\left(\lambda-\lambda_{h}\right) R_{h}(S, T) p \\
& -\lambda_{h} R_{h}(S, T)\left(R_{h}(\mathbf{u}, p)-p\right) .
\end{aligned}
$$

Let us define a function $\delta_{h}$ as

$$
\delta_{h}=R_{h}(\mathbf{u}, p)-p_{h}-r\left(R_{h}(\mathbf{u}, p)-p_{h}, p\right) p .
$$

Since $r(p, p)=1$, it is easy to check that $r\left(R_{h}(\mathbf{u}, p)-p_{h}-r\left(R_{h}(\mathbf{u}, p)-p_{h}, p\right) p, p\right)=0$. Hence the following inequality holds

$$
c\left\|\delta_{h}\right\|_{r} \leq\left\|(I-\lambda T) \delta_{h}\right\|_{r} .
$$

From (3.13), (3.4), (3.2), (3.6), (3.24), (3.26), and $(I-\lambda T) p=0$, we obtain

$$
\begin{aligned}
c\left\|\delta_{h}\right\|_{r} \leq & \left|\lambda_{h}-\lambda\right|\left\|R_{h}(S, T)\left(R_{h}(\mathbf{u}, p)-p_{h}\right)\right\|_{r}+\lambda\left\|\left(R_{h}(S, T)-T\right)\left(R_{h}(\mathbf{u}, p)-p_{h}\right)\right\|_{r} \\
& +\left|\lambda_{h}-\lambda\right|\left\|R_{h}(S, T) p\right\|_{r}+\lambda_{h}\left\|R_{h}(S, T)\left(R_{h}(\mathbf{u}, p)-p\right)\right\|_{r} \\
\leq & C h^{2 s}\left\|T_{h}\left(R_{h}(\mathbf{u}, p)-p_{h}\right)\right\|_{r}+C \lambda h^{\gamma}\left\|T\left(R_{h}(\mathbf{u}, p)-p_{h}\right)\right\|_{1+\gamma} \\
& +C h^{2 s}\left\|T_{h} p\right\|_{r}+\lambda_{h}\left\|R_{h}(S, T)\left(R_{h}(\mathbf{u}, p)-p\right)\right\|_{r} \\
\leq & C h^{2 s}\left\|R_{h}(\mathbf{u}, p)-p_{h}\right\|_{r}+C \lambda h^{\gamma}\left\|R_{h}(\mathbf{u}, p)-p_{h}\right\|_{r} \\
& +C h^{2 s}\|p\|_{r}+\lambda_{h}\left\|R_{h}(S, T)\left(R_{h}(\mathbf{u}, p)-p\right)\right\|_{r} \\
\leq & C h^{2 s}\|p\|_{r}+C \lambda h^{\gamma}\left\|R_{h}(\mathbf{u}, p)-p_{h}\right\|_{r}+\lambda_{h}\left\|R_{h}(S, T)\left(R_{h}(\mathbf{u}, p)-p\right)\right\|_{r},
\end{aligned}
$$

where we used the error estimate

$$
\left\|\left(R_{h}(S, T)-T\right)\left(R_{h}(\mathbf{u}, p)-p_{h}\right)\right\|_{r} \leq C h^{\gamma}\left\|T\left(R_{h}(\mathbf{u}, p)-p_{h}\right)\right\|_{1+\gamma} \leq C h^{\gamma}\left\|R_{h}(\mathbf{u}, p)-p_{h}\right\|_{r},
$$

which is based on the regularity property (3.2).

Now let us estimate the term $\left\|R_{h}(S, T)\left(R_{h}(\mathbf{u}, p)-p\right)\right\|_{r}$. First we choose a function $g_{h} \in W_{h}$ such that $\left\|g_{h}\right\|_{r}=1$ and $-r\left(g_{h}, R_{h}(S, T)\left(R_{h}(\mathbf{u}, p)-p\right)\right)=\left\|R_{h}(S, T)\left(R_{h}(\mathbf{u}, p)-p\right)\right\|_{r}$. Then we define the auxiliary equation: Find $\left(\mathbf{x}_{h}, \xi_{h}\right) \in \mathbf{V}_{h} \times W_{h}$ such that

$$
\left\{\begin{aligned}
a\left(\mathbf{v}_{h}, \mathbf{x}_{h}\right)-b\left(\mathbf{v}_{h}, \xi_{h}\right) & =0, & & \forall \mathbf{v}_{h} \in \mathbf{V}_{h} \\
b\left(\mathbf{x}_{h}, q_{h}\right)+d\left(q_{h}, \xi_{h}\right) & =r\left(g_{h}, q_{h}\right), & & \forall q_{h} \in W_{h}
\end{aligned}\right.
$$


Based on the Babuška-Brezzi condition of the mixed finite element space $\mathbf{V}_{h} \times W_{h}$ and the equivalence between the norms $\|\cdot\|_{r}$ and $\|\cdot\|_{0}$, we have

$$
\begin{aligned}
\left\|\mathbf{x}_{h}\right\|_{\mathbf{v}}+\left\|\xi_{h}\right\|_{0} & \leq C \sup _{0 \neq\left(\mathbf{v}_{h}, q_{h}\right) \in \mathbf{V}_{h} \times W_{h}} \frac{a\left(\mathbf{v}_{h}, \mathbf{x}_{h}\right)-b\left(\mathbf{v}_{h}, \xi_{h}\right)+b\left(\mathbf{x}_{h}, q_{h}\right)+d\left(q_{h}, \xi_{h}\right)}{\left\|\mathbf{v}_{h}\right\|_{\mathbf{v}}+\left\|q_{h}\right\|_{0}} \\
& =C \sup _{0 \neq\left(\mathbf{v}_{h}, q_{h}\right) \in \mathbf{V}_{h} \times W_{h}} \frac{r\left(g_{h}, q_{h}\right)}{\left\|\mathbf{v}_{h}\right\|_{\mathbf{v}}+\left\|q_{h}\right\|_{0}} \\
& \leq C\left\|g_{h}\right\|_{r} \\
& =C .
\end{aligned}
$$

Choosing $\left(\mathbf{v}_{h}, q_{h}\right)=\left(G_{h}(S, T)\left(R_{h}(\mathbf{u}, p)-p\right), R_{h}(S, T)\left(R_{h}(\mathbf{u}, p)-p\right)\right)$ in (3.28) and combining (3.1), (3.3), and (3.5) leads to

$$
\begin{aligned}
-r\left(g_{h}, R_{h}(S, T)\left(R_{h}(\mathbf{u}, p)-p\right)\right)= & a\left(G_{h}(S, T)\left(R_{h}(\mathbf{u}, p)-p\right), \mathbf{x}_{h}\right)-b\left(\mathbf{x}_{h}, R_{h}(S, T)\left(R_{h}(\mathbf{u}, p)-p\right)\right) \\
& -b\left(G_{h}(S, T)\left(R_{h}(\mathbf{u}, p)-p\right), \xi_{h}\right)-d\left(R_{h}(S, T)\left(R_{h}(\mathbf{u}, p)-p\right), \xi_{h}\right) \\
= & a\left(S\left(R_{h}(\mathbf{u}, p)-p\right), \mathbf{x}_{h}\right)-b\left(\mathbf{x}_{h}, T\left(R_{h}(\mathbf{u}, p)-p\right)\right) \\
& -b\left(S\left(R_{h}(\mathbf{u}, p)-p\right), \xi_{h}\right)-d\left(T\left(R_{h}(\mathbf{u}, p)-p\right), \xi_{h}\right) \\
= & -r\left(R_{h}(\mathbf{u}, p)-p, \xi_{h}\right) .
\end{aligned}
$$

Furthermore from the defintion of $J_{h}$, the superconvergence (3.14), and inequality (3.29), we have the following estimate

$$
\begin{aligned}
\left|r\left(R_{h}(\mathbf{u}, p)-p, \xi_{h}\right)\right| & =\left|r\left(R_{h}(\mathbf{u}, p)-J_{h} p, \xi_{h}\right)+r\left(J_{h} p-p, \xi_{h}\right)\right| \\
& \leq\left|\int_{\Omega} \rho\left(J_{h} p-p\right) \xi_{h} \mathrm{~d} \Omega\right|+C h^{s+\gamma}\left\|\xi_{h}\right\|_{0} \\
& =\left|\int_{\Omega} \bar{\rho}\left(J_{h} p-p\right) \xi_{h} \mathrm{~d} \Omega\right|+\left|\int_{\Omega}(\rho-\bar{\rho})\left(J_{h} p-p\right) \xi_{h} \mathrm{~d} \Omega\right|+C h^{s+\gamma}\left\|\xi_{h}\right\|_{0} \\
& \leq C h\|\rho\|_{1, \infty}\left\|J_{h} p-p\right\|_{0}\left\|\xi_{h}\right\|_{0}+C h^{s+\gamma}\left\|\xi_{h}\right\|_{0} \\
& \leq C h^{s+\gamma}\left(1+\|\rho\|_{1, \infty}\right)\left\|\xi_{h}\right\|_{0} \\
& \leq C h^{s+\gamma}\left(1+\|\rho\|_{1, \infty}\right)\left\|g_{h}\right\|_{r},
\end{aligned}
$$

where $\bar{\rho}$ denotes the interplant of $\rho$ onto the piecewise constant function. Then combining (3.30), (3.31), and the definition of $g_{h}$, we obtain

$$
\left\|R_{h}(S, T)\left(R_{h}(\mathbf{u}, p)-p\right)\right\|_{r} \leq C h^{s+\gamma}\left(1+\|\rho\|_{1, \infty}\right) .
$$

So from $(3.25),(3.27)$, and $(3.32)$ and the property of function $\rho$, we can give the estimate for $\left\|R_{h}(\mathbf{u}, p)-p_{h}\right\|_{0}$ as

$$
\begin{aligned}
\left\|R_{h}(\mathbf{u}, p)-p_{h}\right\|_{r} \leq & C\left\|\delta_{h}\right\|_{r}+\left\|r\left(R_{h}(\mathbf{u}, p)-p_{h}, p\right) p\right\|_{r} \\
\leq & C h^{s+\gamma}+C \lambda h^{\gamma}\left\|R_{h}(\mathbf{u}, p)-p_{h}\right\|_{r}+C\left|\left(\rho p, R_{h}(\mathbf{u}, p)-p_{h}\right)\right| \\
= & C h^{s+\gamma}+C \lambda h^{\gamma}\left\|R_{h}(\mathbf{u}, p)-p_{h}\right\|_{r}+C\left|\left(\rho p, R_{h}(\mathbf{u}, p)-p\right)\right| \\
& +C\left|\left(\rho p, p-p_{h}\right)\right| .
\end{aligned}
$$


Now, let us consider the two terms $\left|\left(\rho p, R_{h}(\mathbf{u}, p)-p\right)\right|$ and $\left|\left(\rho p, p-p_{h}\right)\right|$. First from (2.1), (2.8), and (3.13), the following holds

$$
\begin{aligned}
\left|\left(\rho p, p-p_{h}\right)\right| & =\left|(\rho p, p)-\left(\rho p, p_{h}\right)\right| \\
& =\left|\frac{1}{2}(\rho p, p)-\left(\rho p, p_{h}\right)+\frac{1}{2}\left(\rho p_{h}, p_{h}\right)\right| \\
& =\frac{1}{2}\left|\left(\rho\left(p-p_{h}\right), p-p_{h}\right)\right| \\
& \leq C h^{2 s} .
\end{aligned}
$$

Then from (2.4), (3.5), and (3.6), we have

$$
\begin{aligned}
\lambda\left(\rho p, R_{h}(\mathbf{u}, p)-p\right)= & -a\left(\mathbf{u}, G_{h}(\mathbf{u}, p)-\mathbf{u}\right)+b\left(G_{h}(\mathbf{u}, p)-\mathbf{u}, p\right) \\
& +b\left(\mathbf{u}, R_{h}(\mathbf{u}, p)-p\right)+d\left(p, R_{h}(\mathbf{u}, p)-p\right) \\
= & -a\left(\mathbf{u}-G_{h}(\mathbf{u}, p), G_{h}(\mathbf{u}, p)-\mathbf{u}\right)+b\left(G_{h}(\mathbf{u}, p)-\mathbf{u}, p-R_{h}(\mathbf{u}, p)\right) \\
& +b\left(\mathbf{u}-G_{h}(\mathbf{u}, p), R_{h}(\mathbf{u}, p)-p\right)+d\left(p-R_{h}(\mathbf{u}, p), R_{h}(\mathbf{u}, p)-p\right) \\
\leq & C h^{2 s} .
\end{aligned}
$$

Finally combining (3.33), (3.34), and (3.35) leads to

$$
\left\|R_{h}(\mathbf{u}, p)-p_{h}\right\|_{r} \leq C h^{s+\gamma}+C \lambda h^{\gamma}\left\|R_{h}(\mathbf{u}, p)-p_{h}\right\|_{r}
$$

It means when $h$ is small enough, we have

$$
\left\|R_{h}(\mathbf{u}, p)-p_{h}\right\|_{r} \leq C h^{s+\gamma} .
$$

The desired result (3.23) can be obtained by the equivalence between the norms $\|\cdot\|_{r}$ and $\|\cdot\|_{0}$, and the proof is complete.

Based on the obtained superconvergence (3.14) and (3.23), we give some obvious corollaries.

Corollary 3.3. For the eigenfunction approximation $p_{h}$ of the eigenvalue problem (2.4) by the mixed finite element method and the $L^{2}$-projection $J_{h} p$ of the exact eigenfunction, the following superconvergence result holds when the mesh size $h$ is small enough

$$
\left\|p_{h}-J_{h} p\right\|_{0} \leq C h^{s+\gamma} .
$$

Based on this superconvergence result, using a suitable interpolation postprocessing operator for $p_{h}$, we can get a superconvergent eigenfunction approximation.

Corollary 3.4. Let $\left(\mathbf{u}_{h}, p_{h}, \lambda_{h}\right)$ be the corresponding discrete eigenpair approximation for the eigenvalue problem (2.8) and $\left(G_{h}(\mathbf{u}, p), R_{h}(\mathbf{u}, p)\right)$ is the mixed finite element projection of $(\mathbf{u}, p)$ defined by $(3.5)$ where $(\mathbf{u}, p)$ is the exact solution of (2.1). When the mesh size $h$ is small enough, we have the following superconvergence result

$$
\left\|\mathbf{u}_{h}-G_{h}(\mathbf{u}, p)\right\|_{\mathbf{v}}+\left\|R_{h}(\mathbf{u}, p)-p_{h}\right\|_{0} \leq C h^{s+\gamma},
$$

where constant $C$ depends on the eigenvalue $\lambda$ but independent of the mesh size $h$. 
Proof. From (2.4), (2.8), (3.5), (3.38) and Babuška-Brezzi condition, we have

$$
\begin{aligned}
& \left\|\mathbf{u}_{h}-G_{h}(\mathbf{u}, p)\right\|_{\mathbf{v}}+\left\|p_{h}-R_{h}(\mathbf{u}, p)\right\|_{0} \\
& \leq C \sup _{0 \neq\left(\mathbf{v}_{h}, q_{h}\right) \in \mathbf{V}_{h} \times W_{h}} \frac{a\left(\mathbf{u}_{h}-G_{h}(\mathbf{u}, p), \mathbf{v}_{h}\right)-b\left(\mathbf{v}_{h}, p_{h}-R_{h}(\mathbf{u}, p)\right)+b\left(\mathbf{u}_{h}-G_{h}(\mathbf{u}, p), q_{h}\right)+d\left(p_{h}-R_{h}(\mathbf{u}, p), q_{h}\right)}{\left\|\mathbf{v}_{h}\right\|_{\mathbf{v}}+\left\|q_{h}\right\|_{0}} \\
& =C \sup _{0 \neq\left(\mathbf{v}_{h}, q_{h}\right) \in \mathbf{V}_{h} \times W_{h}} \frac{r\left(\lambda_{h} p_{h}-\lambda p, q_{h}\right)}{\left\|\mathbf{v}_{h}\right\|_{\mathbf{v}}+\left\|q_{h}\right\|_{0}} \\
& =C \sup _{0 \neq\left(\mathbf{v}_{h}, q_{h}\right) \in \mathbf{V}_{h} \times W_{h}} \frac{\lambda_{h} r\left(p_{h}-J_{h} p, q_{h}\right)+\lambda_{h} r\left(J_{h} p-p, q_{h}\right)+\left(\lambda_{h}-\lambda\right) r\left(p, q_{h}\right)}{\left\|\mathbf{v}_{h}\right\|_{\mathbf{v}}+\left\|q_{h}\right\|_{0}} \\
& \leq C\left(\left\|p_{h}-J_{h} p\right\|_{0}+h\left\|J_{h} p-p\right\|+\left|\lambda-\lambda_{h}\right|\right) \\
& \leq C h^{s+\gamma}
\end{aligned}
$$

where we use the same technique as in (3.31).

Remark 3.5. From (3.39), we know that there exists supercovergence for both $\left\|G_{h}(\mathbf{u}, p)-\mathbf{u}_{h}\right\|_{\mathbf{V}}$ and $\left\|R_{h}(\mathbf{u}, p)-p_{h}\right\|_{0}$ for general second order elliptic eigenvalue problems by general mixed finite element methods. This is an extension of the results in [11-13].

\section{NumericAl RESUlts}

In this section, we give three numerical examples to illustrate the superconvergence between the mixed finite element approximation $\left(\mathbf{u}_{h}, p_{h}\right)$ and the corresponding mixed finite element projection $\left(G_{h}(\mathbf{u}, p), R_{h}(\mathbf{u}, p)\right)$. Furthermore the superconvergence between $p_{h}$ and interpolant $J_{h} p$ is also investigated here. Throughout this section, numerical results are given for the first eigenvalue and its corresponding eigenfunction. Of course, we should point out that our result is also valid for other eigenvalues. Here, the implicitly restarted Arnoldi method is applied to compute the eigenvalues. The Raviart-Thomas, Brezzi-Douglas-Marini and Brezzi-Douglas-FortinMarini mixed finite elements are applied to solve the eigenvalue problems on the triangular and rectangular meshes. The notation used in the error figures is defined as follows

$$
R_{h} p:=R_{h}(\mathbf{u}, p) \text {, and } G_{h} \mathbf{u}=G_{h}(\mathbf{u}, p),
$$

where $(\mathbf{u}, p)$ is the exact solution of the corresponding eigenvalue problem.

\subsection{Model eigenvalue problem}

In this subsection, we investigate the superconvergence phenomena of the model eigenvalue problem

$$
\left\{\begin{aligned}
-\Delta p & =\lambda p, & & \text { in } \Omega \\
p & =0, & & \text { on } \partial \Omega, \\
\int_{\Omega} p^{2} \mathrm{~d} \Omega & =1, & &
\end{aligned}\right.
$$

on the domain $\Omega=(0,1) \times(0,1)$.

It is well known that the exact eigenpair is

$$
p_{k l}=\sin (k \pi x) \sin (l \pi y), \quad \lambda_{k l}=\left(k^{2}+l^{2}\right) \pi^{2}, \quad 0 \leq k, l \text { and } k, l \text { are integers. }
$$

Here we test the superconvergence property on triangular and rectangular meshes. On the triangular meshes, the lowest and first order Raviart-Thomas $\left(R T_{0}\right.$ and $\left.R T_{1}\right)$ and the lowest order Brezzi-Douglas-Marini $\left(B D M_{1}\right)$ mixed finite elements are applied to do the test. Additionally we also use the lowest and first order RaviartThomas $\left(R T_{0}\right.$ and $\left.R T_{1}\right)$, the first order Brezzi-Douglas-Fortin-Marini $\left(B D F M_{1}\right)$ mixed finite elements on the rectangular meshes to solve the eigenvalue problem (4.1). 

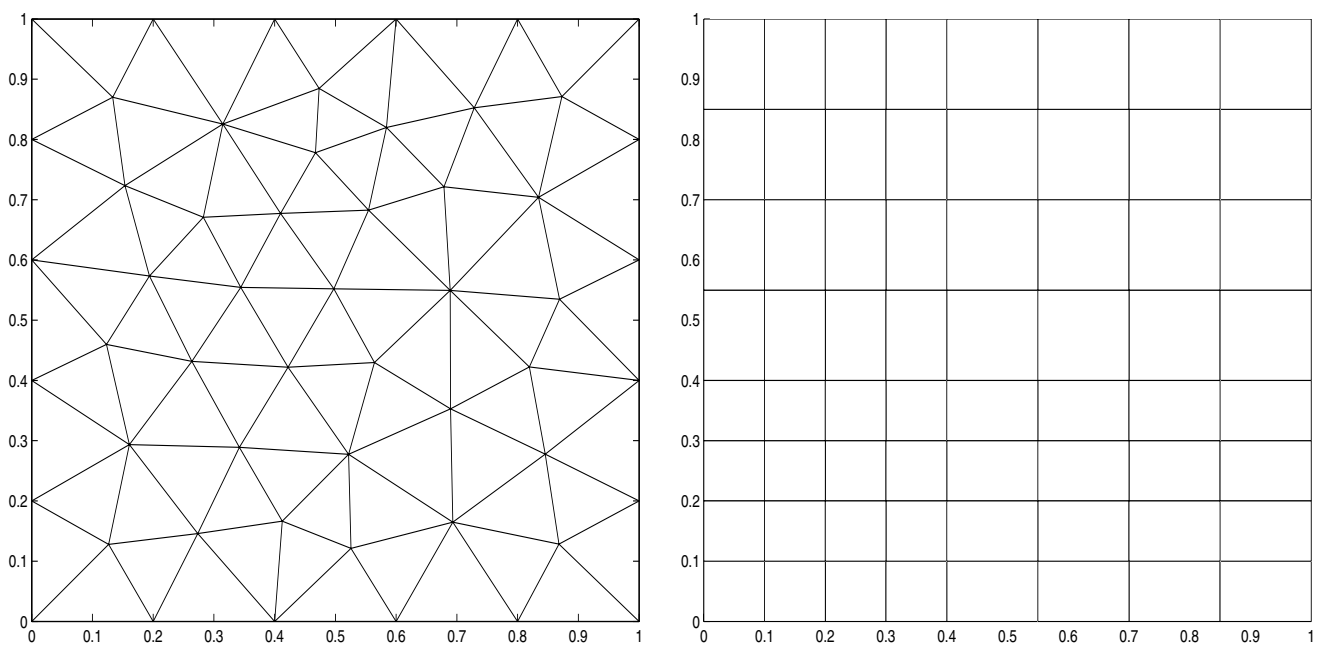

FiguRE 1. Initial triangular and rectangular meshes for regular refinement.
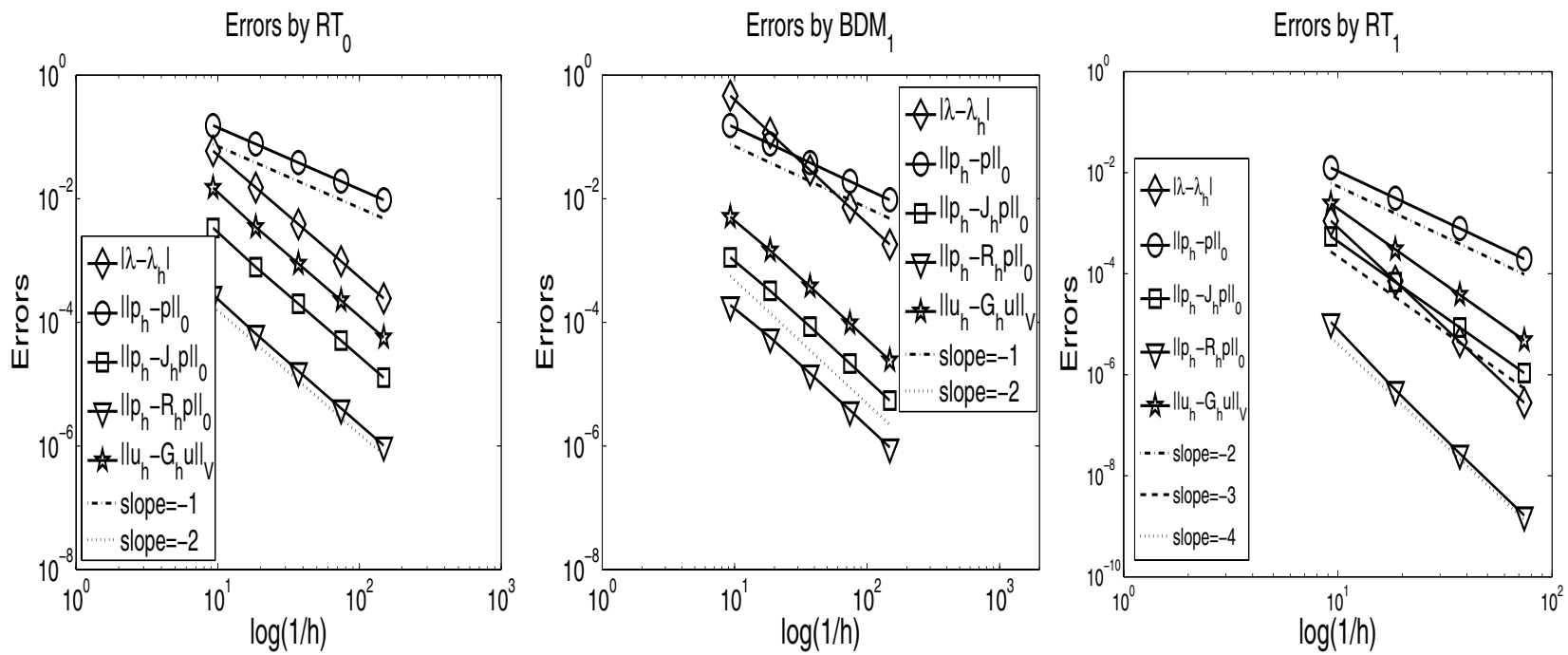

FiguRE 2. Errors of $R T_{0}, B D M_{1}$ and $R T_{1}$ on the regular refinement triangular meshes for model problem.

First we give the numerical results of the superconvergence on the triangular meshes obtained by regular refinement of the initial mesh showed in Figure 1. The corresponding numerical results with $R T_{0}, B D M_{1}$ and $R T_{1}$ are presented in Figure 2. We also test the $R T_{0}, R T_{1}$ and $B D F M_{1}$ mixed finite elements on the rectangular meshes. Right hand side of Figure 1 shows the initial mesh and we use regular refinement to produce the mesh sequence. The numerical results are showed in Figure 3.

In order to test the superconvergence on more general meshes. We also test the superconvergence on the mesh sequence in which each level triangular mesh is obtained by Delaunay algorithm. The triangular mesh sequence is presented in Figure 4. The corresponding numerical results are listed in Figure 5. 

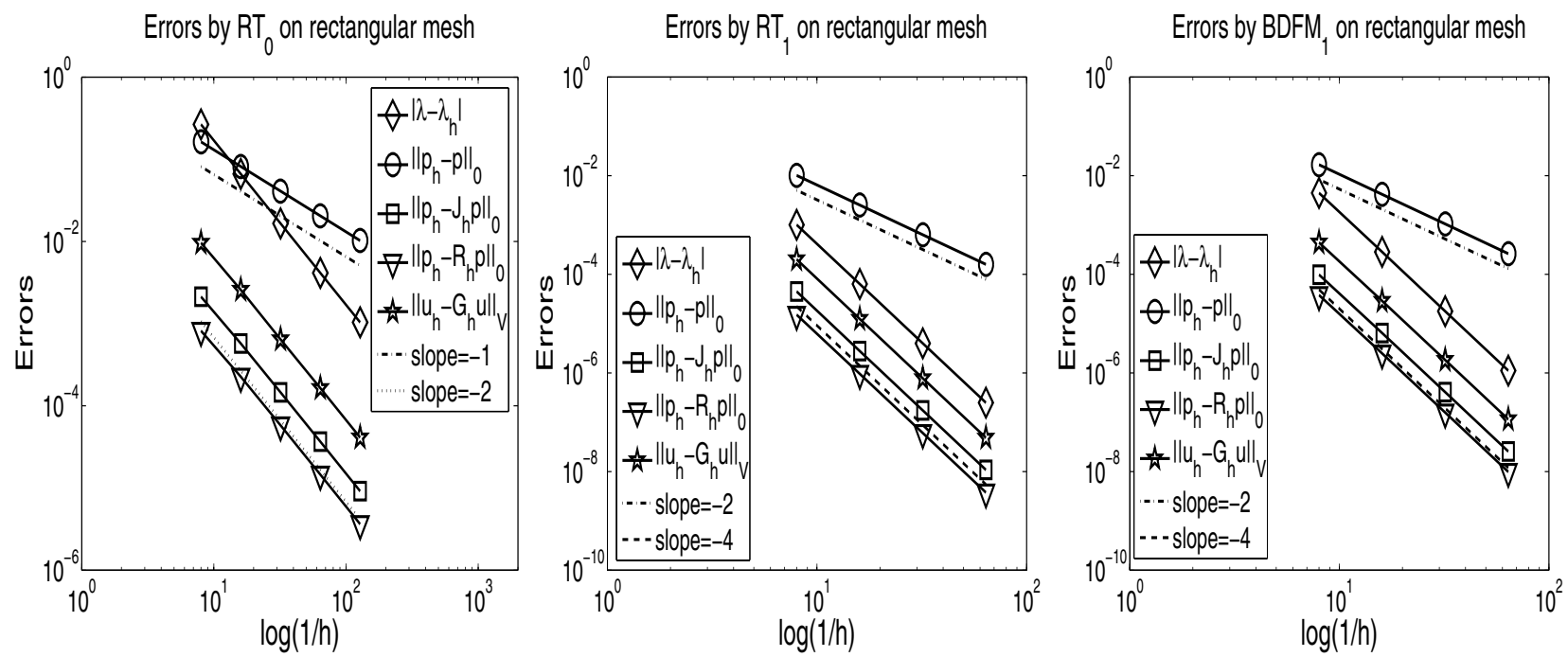

FiguRE 3. Errors of $R T_{0}, R T_{1}$ and $B D F M_{1}$ on the regular refinement rectangular meshes for model problem.
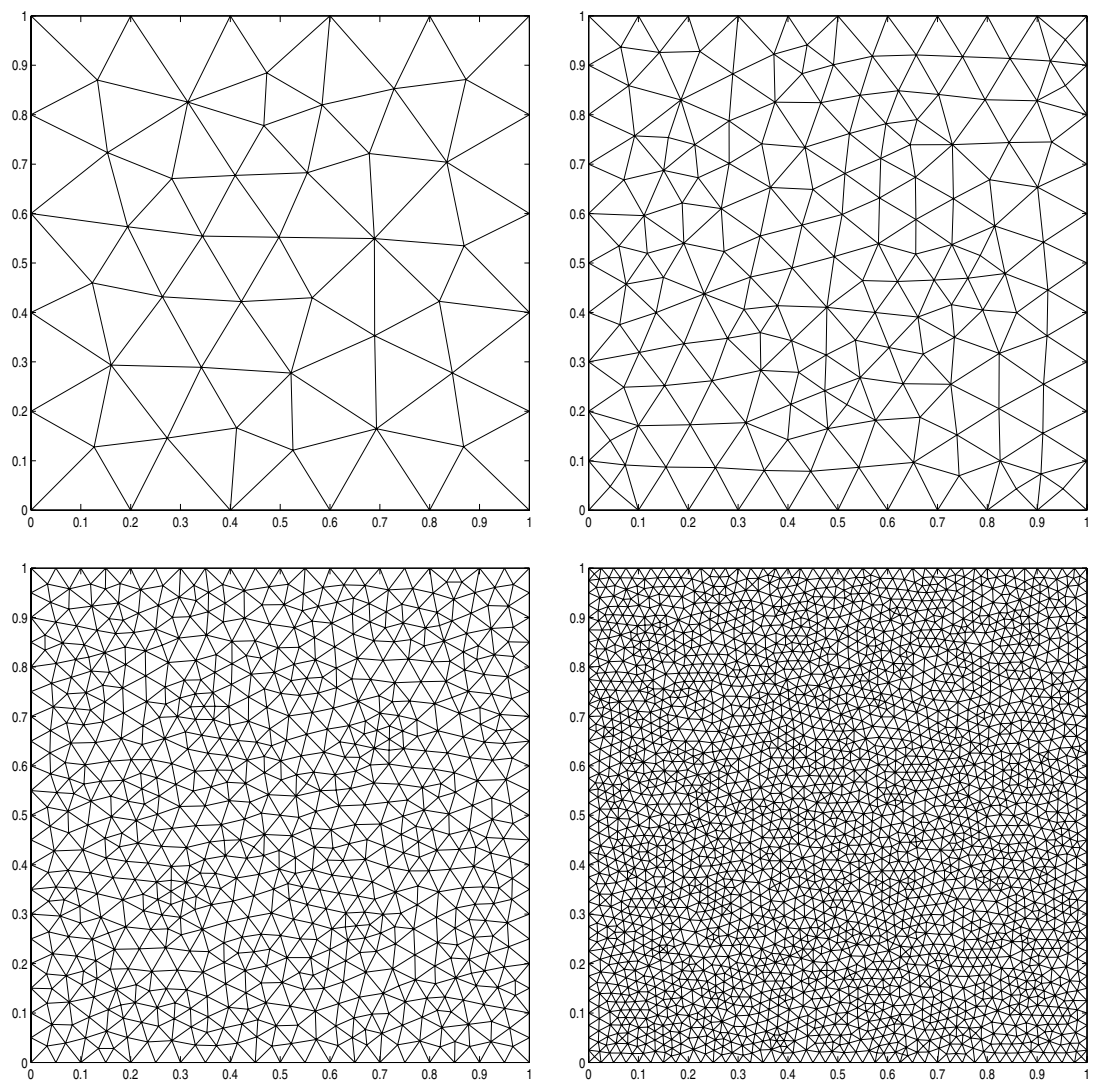

FigURE 4. Unstructured triangular mesh sequence. 

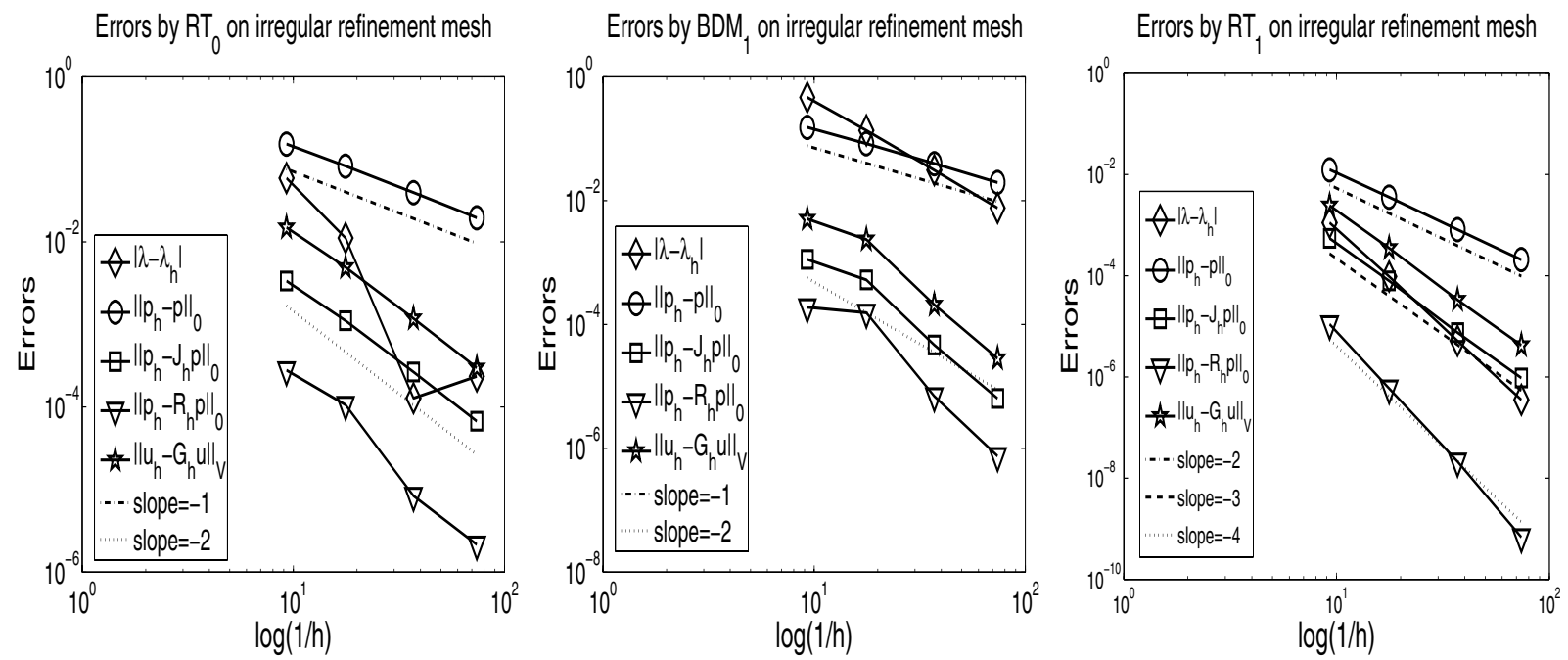

FiguRE 5. Errors of $R T_{0}, B D M_{1}$ and $R T_{1}$ on the irregular refinement triangular meshes for model problem.

From Figures 2, 3, and 5, we can find that there exists superconvergence for the eigenfunction approximation $\left(\mathbf{u}_{h}, p_{h}\right)$ and the corresponding projection $\left(G_{h}(\mathbf{u}, p), R_{h}(\mathbf{u}, p)\right)$, the approximation $p_{h}$ and interpolant $J_{h} p$. This confirms the results of Corollaries 3.3 and 3.4.

\subsection{More general eigenvalue problem}

In this subsection, we are concerned with a more general eigenvalue problem (1.1) with Dirichlet boundary condition (1.2), and

$$
\mathcal{A}=\left(\begin{array}{cc}
1+\left(x_{1}-\frac{1}{2}\right)^{2} & \left(x_{1}-\frac{1}{2}\right)\left(x_{2}-\frac{1}{2}\right) \\
\left(x_{1}-\frac{1}{2}\right)\left(x_{2}-\frac{1}{2}\right) & 1+\left(x_{2}-\frac{1}{2}\right)^{2}
\end{array}\right),
$$

$\varphi=e^{\left(x_{1}-\frac{1}{2}\right)\left(x_{2}-\frac{1}{2}\right)}, \rho=1+\left(x_{1}-\frac{1}{2}\right)\left(x_{2}-\frac{1}{2}\right)$ and $\Omega=(0,1) \times(0,1)$.

Since the exact solution is not known, we choose an adequately accurate approximation $\lambda=23.7784248452082$ which is obtained by extrapolation method (see, e.g., $[17,19]$ ) as the exact first eigenvalue (has 8 significant digits at least). In order to measure the errors of eigenfunction approximations, we use the corresponding higher order Galerkin finite element eigenfunction approximation, which has higher order accuracy in $L^{2}(\Omega)$ norm sense, as the exact eigenfunction.

First we test the superconvergence on triangular meshes obtained by the regular refinement with the initial mesh showed in Figure 1. The corresponding numerical results by $R T_{0}, B D M_{1}$, and $R T_{1}$ mixed finite elements are presented in Figure 6. Then we give the numerical results of $R T_{0}, R T_{1}$ and $B D F M_{1}$ mixed finite elements on rectangular meshes which are also obtained by regular refinement with the initial mesh showed in Figure 1. Figure 7 shows the corresponding numerical results by $R T_{0}, R T_{1}$, and $B D F M_{1}$.

From Figures 6 and 7, we can find there also exists superconvergence of the general problem and this confirms the results of Corollaries 3.3 and 3.4 again.

Remark 4.1. From Figures 2, 3, 5, 6, and 7, it seems that there exists the ultrasuperconvergence $\left(\| p_{h}-\right.$ $\left.R_{h}(\mathbf{u}, p) \|_{0} \leq C h^{s+2}\right)$ for $p_{h}-R_{h}(\mathbf{u}, p)$ by high order mixed finite element method. The reason may rest with $\gamma=2$ for high order mixed finite element methods on the unit square domain. 

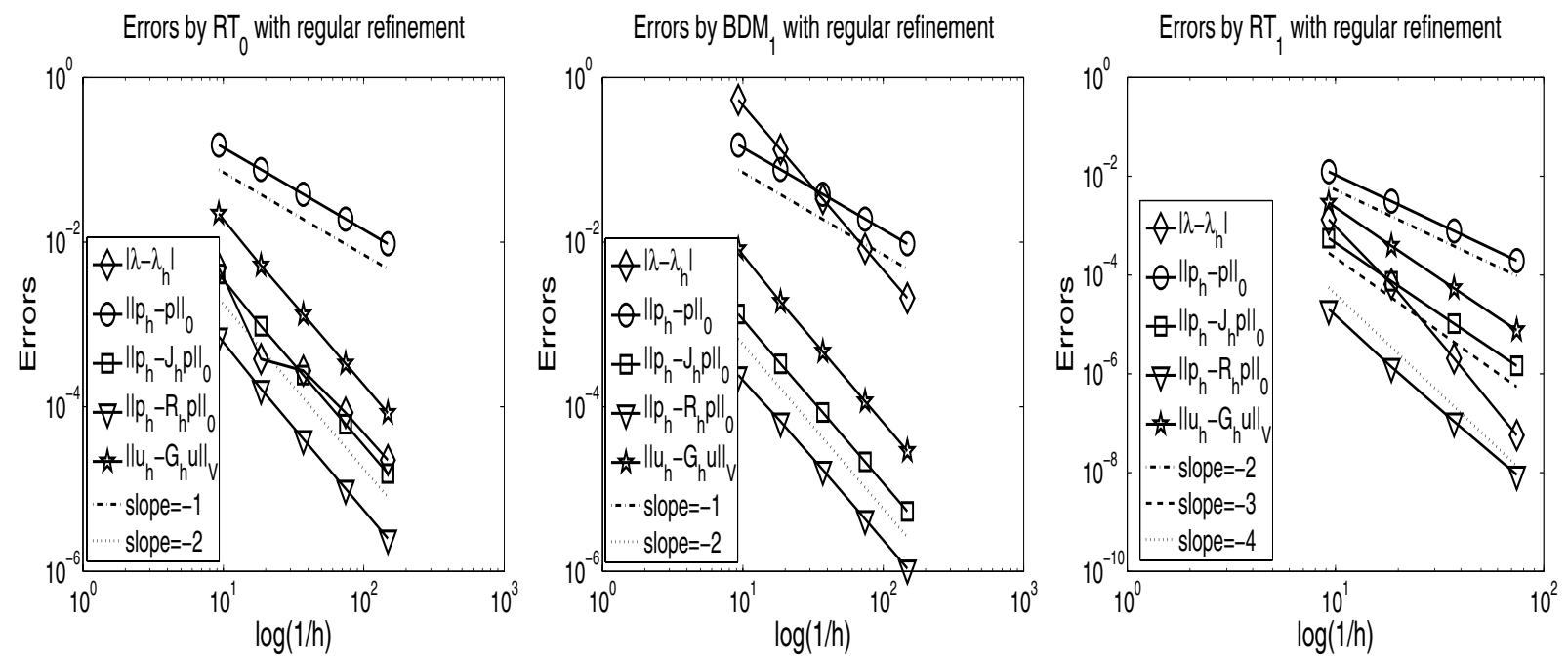

FiguRE 6 . Errors of $R T_{0}, B D M_{1}$ and $R T_{1}$ on the regular refinement triangular meshes for general problem.
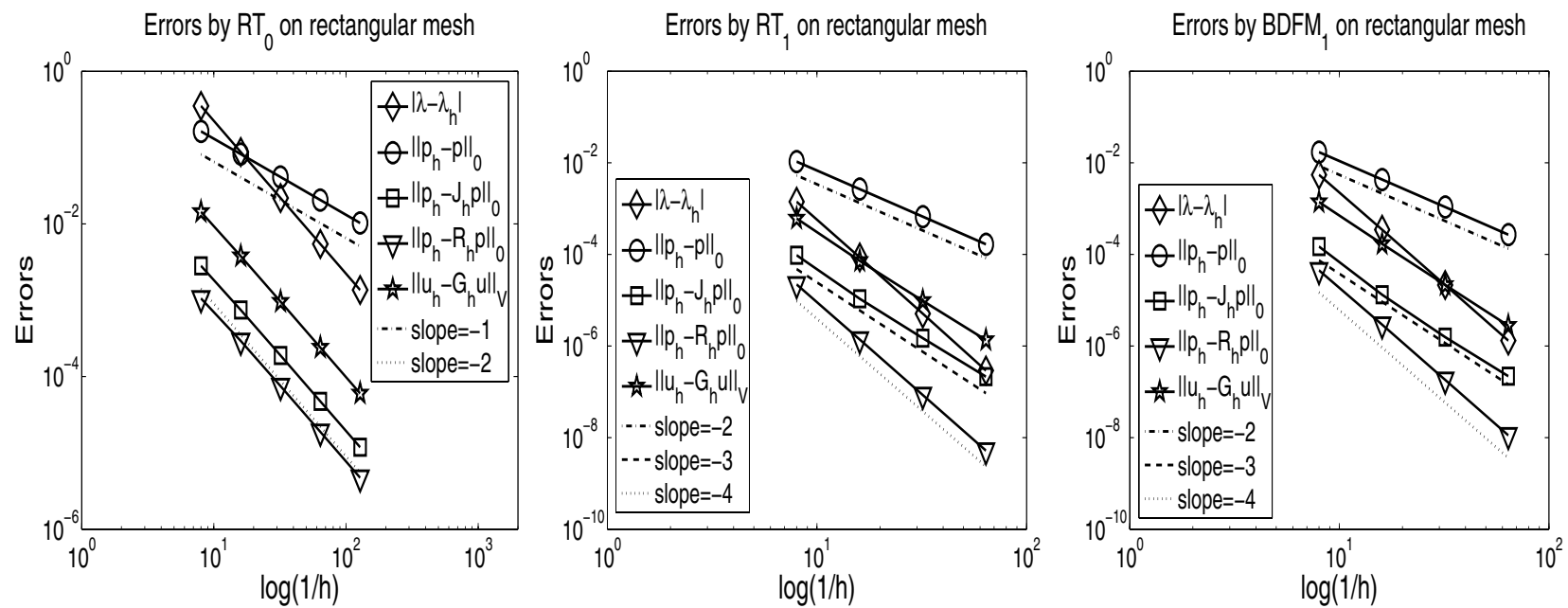

FIgURE 7. Errors of $R T_{0}, R T_{1}$ and $B D F M_{1}$ on the regular refinement rectangular meshes for general problem.

\section{3. $L$ shape domain}

Here we solve the model problem (4.1) but with $L$ shape domain $\Omega:=(-1,1) \times(-1,1) \backslash[-1,0] \times[0,1]$. It is known that the eigenfunction corresponding to the first eigenvalue is singular.

We solve this problem with $R T_{0}$ and $B D M_{1}$ mixed finite elements on triangular meshes, which are obtained by regular refinement with the initial mesh showed in Figure 8. The same as last subsection, the first exact eigenvalue and the corresponding eigenfunction is not known neither. We adopt an adequately accurate approximation $\lambda=9.6397238440219$ (see, e.g., [17]) as the smallest accurate eigenvalue and the eigenfunction obtained by higher order Galerkin finite element method as the accurate eigenfunction according to the first eigenvalue. The numerical results are listed in Figure 9. 


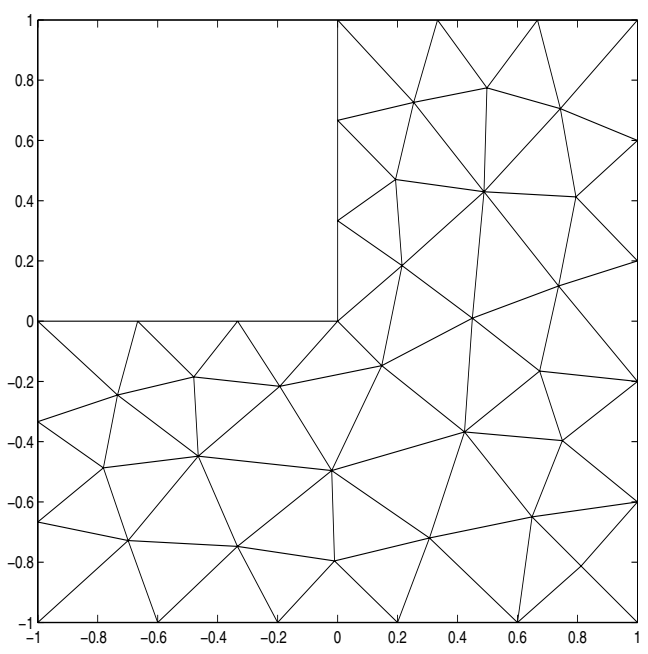

FiguRE 8. Initial mesh for $L$ shape domain.
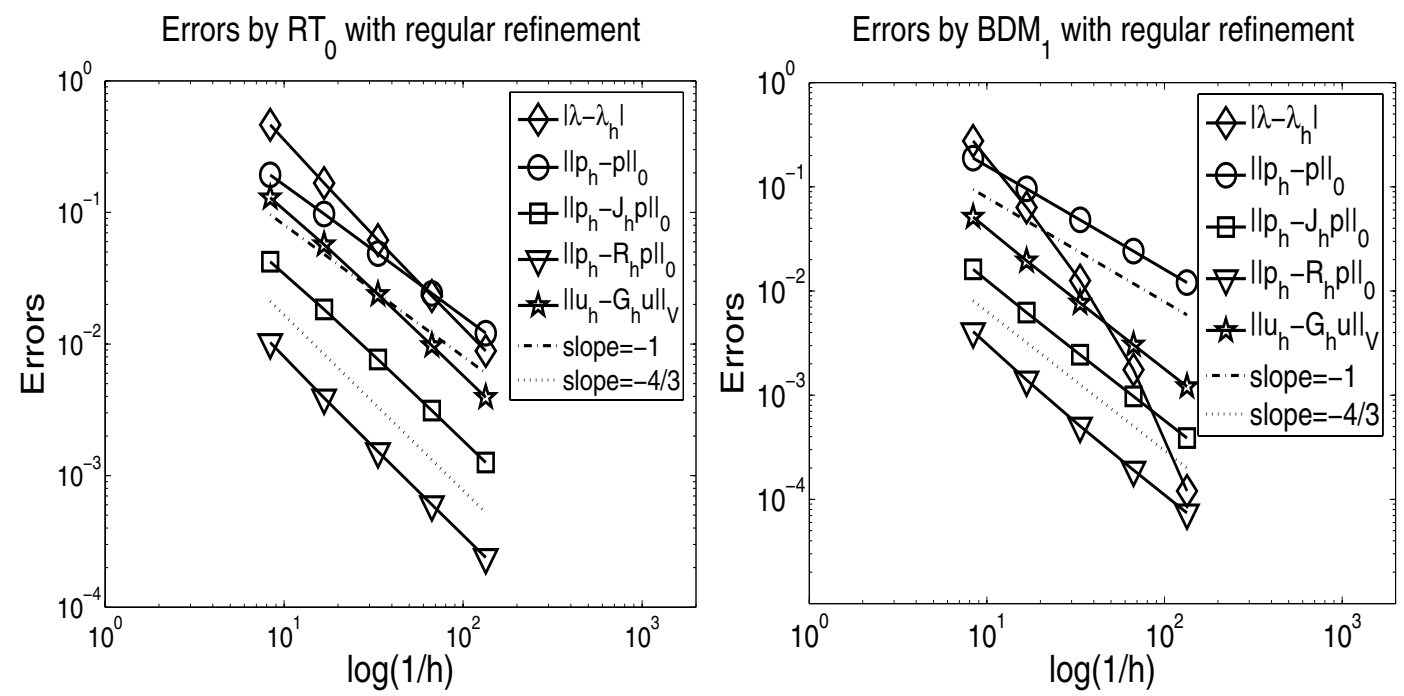

FiguRE 9. Errors of $R T_{0}$ and $B D M_{1}$ on the regular refinement triangular meshes for $L$ shape domain problem.

Figure 9 shows that there exists superconvergence between the eigenfunction approximation $\left(\mathbf{u}_{h}, p_{h}\right)$ and the corresponding projection $\left(G_{h}(\mathbf{u}, p), R_{h}(\mathbf{u}, p)\right)$, the eigenfunction approximation $p_{h}$ and the interpolant $J_{h} p$ even for the singular eigenfunction. This confirms Corollaries 3.3 and 3.4.

Remark 4.2. Figure 9 shows that the convergence order of $\left\|p_{h}-p\right\|_{0}$ is 1 which is larger than our expectancy. The reason is that the eigenfunction $p \in H^{5 / 3-\epsilon}(\Omega)$ on the $L$-shape domain, the superconvergence result (3.38) and the following inequality

$$
\left\|p-p_{h}\right\|_{0} \leq\left\|p-J_{h} p\right\|_{0}+\left\|J_{h} p-p_{h}\right\|_{0} \leq C h\|p\|_{1}+C h^{4 / 3} \leq C h .
$$


The surprising convergence rate of the eigenvalue approximation by $B D M_{1}$ element may due to the inexactness of our used "accurate" eigenvalue $\lambda=9.6397238440219$ in this example.

\section{Concluding Remarks}

In this paper, we give a superconvergence result for the general second order elliptic eigenvalue problems by mixed finite element methods. Based on the results in Corollaries 3.3 and 3.4, as discussed in the superconvergence theory $[16,18]$, suitable interpolation postprocessing methods can be applied to improve the convergence order of the eigenfunction approximations. Additionly, based on the result of Corollary 3.4, we can also obtain some superconvergence results on some special types of structured meshes with the integral identity techniques in superconvergence theory (see, e.g., $[16,18]$ ). Furthermore, with this superconvergence result, the Rayleigh quotient acceleration technique can be applied to improve the accuracy of the eigenvalue approximations. These will be our future works.

Acknowledgements. The authors wish to thank the two referees for their constructive criticism and the valuable comments; they led to a significant improvement in the presentation of our paper.

\section{REFERENCES}

[1] I. Babuška and J.E. Osborn, Finite element-Galerkin approximation of the eigenvalues and eigenvectors of selfadjoint problems. Math. Comp. 52 (1989) 275-297.

[2] I. Babuška and J. Osborn, Eigenvalue Problems, in Handbook of Numerical Analysis II, Finite Element Methods (Part 1), edited by P.G. Lions and P.G. Ciarlet. North-Holland, Amsterdam (1991) 641-787.

[3] C. Bacuta and J.H. Bramble, Regularity estimates for the solutions of the equations of linear elasticity in convex plane polygonal domain, Special issue dedicated to Lawrence E. Payne. Z. Angew. Math. Phys. 54 (2003) 874-878.

[4] D. Boffi, Finite element approximation of eigenvalue problems. Acta Numer. 19 (2010) 1-120.

[5] D. Boffi, F. Brezzi and L. Gastaldi, On the convergence of eigenvalues for mixed fomulations. Ann. Scuola Norm. Sup. Pisa Cl. Sci 25 (1997) 131-154.

[6] D. Boffi, F. Brezzi and L. Gastaldi, On the problem of spurious eigenvalues in the approximation of linear elliptic problems in mixed form. Math. Comp. 69 (2000) 121-140.

[7] S. Brenner and L. Scott, The Mathematical Theory of Finite Element Methods. Springer-Verlag, New York (1994).

[8] F. Brezzi and M. Fortin, Mixed and Hybrid Finite Element Methods. Springer-Verlag, New York (1991).

[9] F. Chatelin, Spectral Approximation of Linear Operators. Academic Press Inc., New York (1983).

[10] J. Douglas and J.E. Roberts, Global estimates for mixed methods for second order elliptic equations. Math. Comp. 44 (1985) $39-52$.

[11] R. Durán, L. Gastaldi and C. Padra, A posteriori error estimators for mixed approximations of eigenvalue problems. Math. Models Methods Appl. Sci. 9 (1999) 1165-1178.

[12] F. Gardini, A posteriori error estimates for an eigenvalue problem arising from fluid-structure interaction. Instituto Lombardo (Rend. Sc.) (2004) 138.

[13] F. Gardini, Mixed approximation of eigenvalue problems: a superconvergence result. ESAIM: M2AN 43 (2009) 853-865.

[14] V. Girault and P. Raviart, Finite Element Methods for Navier-Stokes Equations, Theory and Algorithms. Springer-Verlag, Berlin (1986).

[15] P. Grisvard, Singularities in Boundary Problems. MASSON and Springer-Verlag (1985).

[16] Q. Lin and J. Lin, Finite Element Methods: Accuracy and Inprovement. China Sci. Tech. Press (2005).

[17] Q. Lin and H. Xie, Asymptotic error expansion and Richardson extrapolation of eigenvalue approximations for second order elliptic problems by the mixed finite element method. Appl. Numer. Math. 59 (2009) 1884-1893.

[18] Q. Lin and N. Yan, The Construction and Analysis of High Efficiency Finite Element Methods. HeBei University Publishers (1995) (in Chinese)

[19] Q. Lin, H. Huang and Z. Li, New expansion of numerical eigenvalue for $-\Delta u=\lambda \rho u$ by nonconforming elements. Math. Comp. 77 (2008) 2061-2084.

[20] B. Mercier, J. Osborn, J. Rappaz and P.A. Raviart, Eigenvalue approximation by mixed and hybrid methods. Math. Comp. 36 (1981) 427-453.

[21] J. Osborn, Approximation of the eigenvalue of a nonselfadjoint operator arising in the study of the stability of stationary solutions of the Navier-Stokes equations. SIAM J. Numer. Anal. 13 (1976) 185-197. 